\title{
Radiologisch-interventionelle Verfahren zur Therapie von kolorektalen Lebermetastasen
}

\author{
Interventional Radiological Procedures in the Therapy for \\ Colorectal Liver Metastases
}

Autoren

Institut
R. Damm*, R. Seidensticker*, J. Ricke, M. Seidensticker

Klinik für Radiologie und Nuklearmedizin, Universitätsklinikum Magdeburg A. ö.R., Magdeburg, Deutschland

\author{
Schlüsselwörter \\ - interventionelle Radiologie \\ - Lebermetastase \\ - Ablationstechniken \\ - minimalinvasive Chirurgie \\ - Radioonkologie \\ Key words \\ - interventional radiology \\ - liver metastases \\ - ablation techniques \\ - minimally invasive surgical \\ procedures \\ - radiation oncology
}

\section{Zusammenfassung \\ $\nabla$}

Mikrotherapeutische Verfahren in der interventionellen Radiologie stellen bei Inoperabilität (technische Inoperabilität, funktionelle Inoperabilität, Komorbiditäten, Wunsch des Patienten) neben der Chemotherapie eine Möglichkeit zur lokalen Kontrolle von Lebermetastasen dar. Einen Schwerpunkt stellen lokale Verfahren wie die Radiofrequenzablation oder die interstitielle Brachytherapie dar, bei denen unter Bildführung durch Ultraschall sowie CT- oder offene MRT-Systeme eine thermische bzw. radiogene Ablation der Malignome durchgeführt wird. Diese erweisen sich als sehr effektiv, sind dabei aber auf einen oligonodulären Befall limitiert. Zur Behandlung einer disseminierten Metastasierung haben sich lokoregionäre Techniken wie die Yttrium-90Radioembolisation etabliert. Hier wird unter angiografischer Kontrolle der Wirkstoff in Form von radioaktiv markierten Mikrosphären über einen arteriellen Zugang appliziert. Dieser Artikel fokussiert sich auf Metastasen des kolorektalen Karzinoms als häufigste Tumorentität zur interventionell-radiologischen Therapie.

\section{Einführung \\ $\nabla$}

Zentralbl Chir 2013; 138

76-83 ๑ Georg Thieme

Verlag KG Stuttgart · New York ·

ISSN 0044-409X

\section{Korrespondenzadresse}

Dr. Max Seidensticker

Universitätsklinikum

Magdeburg A. ö.R.

Klinik für Radiologie und

Nuklearmedizin

Leipziger Str. 44

39120 Magdeburg

Deutschland

Tel.: 0391/67-13030

Fax: 0391/67-13029

max.seidensticker@

med.ovgu.de
Die Leber ist das häufigste Zielorgan einer hämatogenen, portalvenösen Metastasierung von gastrointestinalen Tumoren.

Dabei entstammt etwa die Hälfte der Lebermetastasen einem kolorektalen Karzinom (CRC). In vielen Fällen wird das Überleben durch die hepatische Metastasierung begrenzt; bei hepatisch metastasierten kolorektalen Karzinomen im natürlichen Verlauf finden sich exemplarisch über alle Stadien hinweg 1-Jahres-Überlebensraten

* Robert Damm und Ricarda Seidensticker haben gleichen Anteil an der Erstautorenschaft.

\section{Abstract \\ $\nabla$}

Microtherapeutic procedures performed by interventional radiologists pose a viable alternative or additive to systemic chemotherapy for local tumour control in cases of non-operable (for technical, functional, and comorbidity reasons or at the patient's wish) liver metastases. A main focus includes local therapies such as radiofrequency ablation and interstitial brachytherapy which are performed under ultrasound, CT or MRI guidance to achieve a thermal or radiogenic ablation of the malignancy. Although highly effective, these procedures are limited to oligonodular manifestations. For disseminated metastases, locoregional techniques like the yttrium-90 radioembolisation have become established. Here, the active principle in the form of radioactively labelled microspheres is introduced into the liver through an arterial catheter under angiographic guidance. The present article focuses on metastases of colorectal cancer as the most frequent tumour entity encountered in interventional radiotherapy.

bei lediglich einem Drittel der Patienten. Bei disseminiertem Befall (hepatisch und extrahepatisch) ergeben sich ohne Therapie noch deutlich geringere Überlebenszeiten [1,2].

Als bislang einziges kuratives Verfahren gilt die chirurgische Resektion der Lebermetastasen, obwohl für bestimmte mikrotherapeutische Verfahren ein kuratives Potenzial diskutiert wird [3]. In potenziell kurativer Intention oder zum Erreichen einer Langzeitremission wird eine R0-Resektion angestrebt. Durch Resektion der Lebermetastasen kann ein 5-Jahres-Überleben zwischen 28 und $48 \%$ der Patienten erreicht werden [4]. 


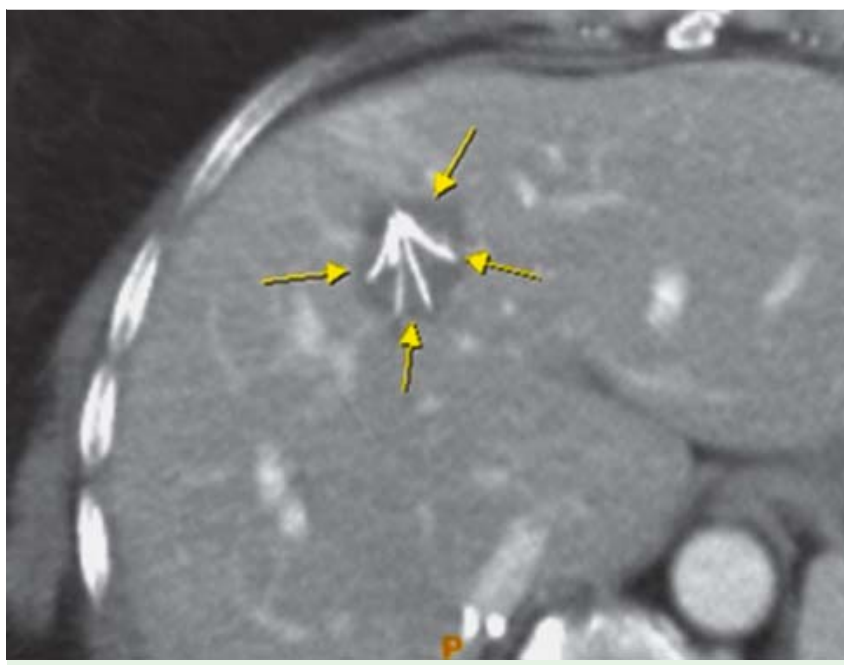

Abb. 1 Periinterventionelles CT: hypodens demarkierte Lebermetastase (Pfeile) mit einliegender Radiofrequenzsonde.

Nur für ein hoch selektioniertes Patientengut ermöglichen wiederholte Resektionen in der retrospektiven Auswertung ein 5Jahres-Überleben von 73\% [5].

Viele Patienten mit hepatisch metastasiertem CRC zeigen jedoch ein intra- oder extrahepatisches Rezidiv im Verlauf nach R0-Resektion. Der initial kurative Therapieansatz kann somit nur für einen selektierten Anteil der Patienten dauerhaft erreicht werden [6].

Im Falle einer Irresektabilität (ca. $2 / 3$ der Patienten mit kolorektalen Lebermetastasen) steht oftmals die systemische Chemotherapie im Vordergrund der Therapiebemühungen. Für das metastasierte kolorektale Karzinom ist die Therapie mit einer Kombination aus 5-Fluorouracil und Folinsäure mit Oxaliplatin oder Irinotecan der derzeitige Standard, der gegebenenfalls um Antikörper wie Bevacizumab ergänzt werden kann. Hiermit kann ein medianes Überleben von über 20 Monaten erreicht werden [7].

Als weiterer Zweig in der interdisziplinären Tumortherapie bieten die radiologisch-interventionellen Verfahren zahlreiche Möglichkeiten zur Tumorkontrolle in der Leber und können somit zur Verlängerung des Überlebens beitragen [8]. Speziell Patienten, die die Kriterien für eine Metastasenresektion initial oder im Verlauf nicht erfüllen oder deren Allgemeinzustand die weitere Anwendung von Zytostatika limitiert, können von den durch niedrige Mortalität und Morbidität charakterisierten minimalinvasiven Verfahren profitieren [9].

Aufgrund der allgemeinen Verbreitung wird in diesem Artikel als Entität das kolorektale Karzinom thematisiert. Dabei möchte dieser Artikel den Lesern unter den zahlreichen radiologischen Verfahren die Radiofrequenzablation (RFA), weitere lokale Verfahren wie die laserinduzierte Thermotherapie (LITT), die interstitielle Brachytherapie sowie die Radioembolisation (RE) vorstellen und die vorliegenden Studienergebnisse ( Tab. 1) kritisch diskutieren. Erwähnung finden ebenfalls die Nebenwirkungen dieser Therapien.

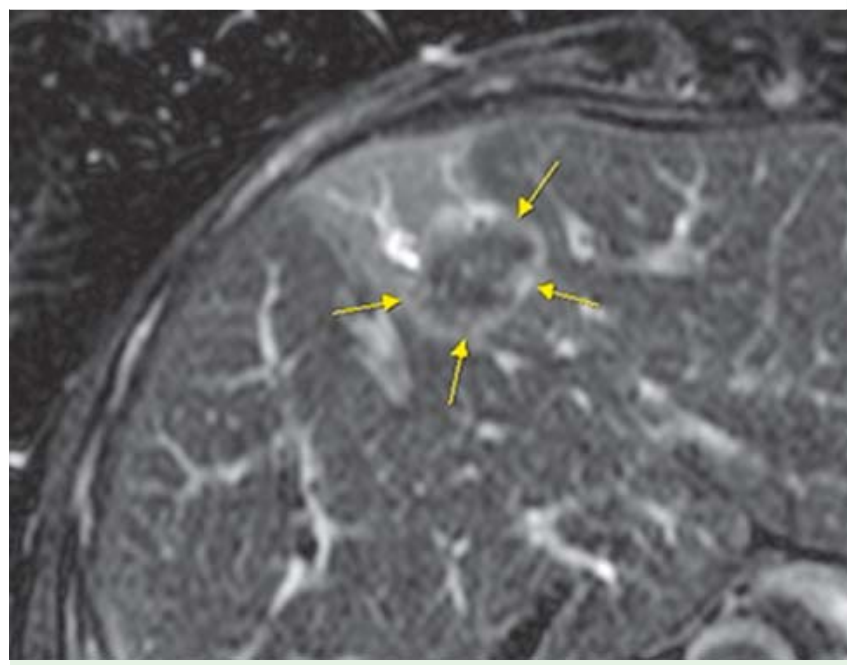

Abb. 2 Postinterventionelles MRT 3 Monate nach RFA: Residuelle Narbe (Pfeile) mit nachgeschalteter Begleitreaktion und leichtem perifokalen Ödem, angrenzend in der Peripherie leicht erweiterter Gallengang. In der weiteren Nachsorge zeigte sich eine anhaltende Remission (T2w mit Fettsuppression).

\section{Lokale Verfahren \\ 7}

\section{Radiofrequenzablation}

Die Radiofrequenzablation (RFA) ist einerseits als intraoperativ eingesetztes Verfahren etabliert und stellt andererseits das aktuell verbreitetste radiologisch-interventionelle Verfahren zur perkutanen Ablation von Lebermetastasen dar. In beiden Anwendungen wird äquivalent zur chirurgischen Resektion eine vollständige Eliminierung der Tumorzellen angestrebt.

Bei der RFA wird mittels Applikation von Wechselströmen im Gewebe eine Hyperthermie mit folgender Koagulationsnekrose erzeugt, die auf eine Größe von bis zu $5 \mathrm{~cm}$ ausgedehnt werden kann, wobei durch Umpositionierung der Nadel eine größere Ablationszone erreicht werden kann [28]. Zur kompletten Ablation wird ein Sicherheitssaum von $1 \mathrm{~cm}$ um den Tumor eingeschlossen. Die Deutsche Gesellschaft für Interventionelle Radiologie und minimal-invasive Therapie empfiehlt einen maximalen Tumordurchmesser von $3,5 \mathrm{~cm}$ zur sicheren Ablation bei multifokaler Metastasierung sowie bei einem unifokalen Herd einen maximalen Durchmesser von $5 \mathrm{~cm}$ mit $7 \mathrm{~cm}$ messender Ablationszone [29]. Um mit einer Resektion vergleichbare Ergebnisse zu erreichen, ist nach aktuellen Studien eine maximale Metastasengröße von $3 \mathrm{~cm}$ empfehlenswert [30].

Limitationen des Verfahrens sind vor allem Kühlungseffekte durch benachbarte Gefäße, die begrenzte Größe der induzierten Koagulationsnekrose und die Hitzevulnerabilität angrenzender Strukturen (Gallengänge etc.).

Die RFA kann perkutan sowohl unter sonografischer Bildführung als auch CT- oder MRT-gestützt angewendet werden. Nachteil der Sonografie ist hierbei die Bildempfindlichkeit gegenüber der wärmebedingten Gasbildung im Verlauf der Ablation und die schlechte Darstellbarkeit tieferer Leberabschnitte [31]. Hier zeigt sich ein Vorteil der fluoroskopischen Nadelplatzierung und Ablation in der Computertomografie [32]. Die Anwendung in der offenen MRT bietet darüber hinaus das simultane Monitoring der Temperaturentwicklung und die multiplanare Darstellung der Applikatoren, die eine optimale Platzierung erlaubt [33]. Ein Beispiel einer bildgeführten Radiofrequenzablation und Nachsorge 3 


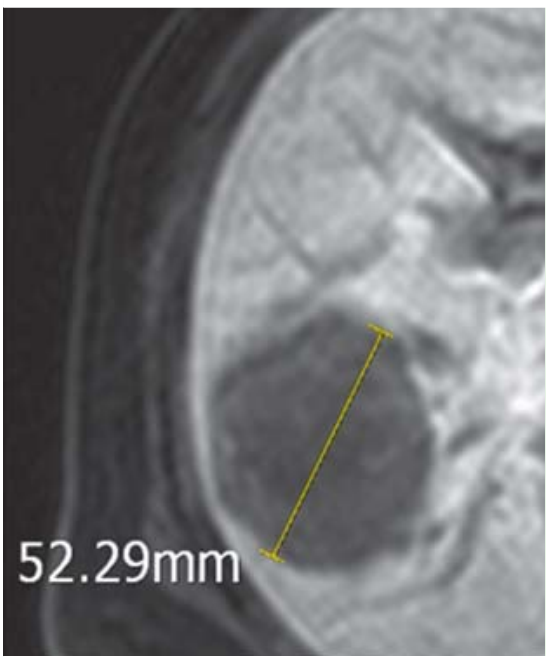

Abb. 3 MRT vor interstitieller Brachytherapie: Metastase eines kolorektalen Karzinoms $>5 \mathrm{~cm}$ (T1w GRE mit leberspezifischem Kontrastmittel Gd-EOBDTPA in der hepatobiliären Phase 20 min nach Applikation).

Monate postinterventionell findet sich in Abb. 1 sowie - Abb. 2.

Die klinische Anwendung ist bisher in vielen Studien evaluiert, jedoch finden sich häufig monozentrische Erfahrungen ohne Vergleichsarme. Darüber hinaus ist eine Stratifizierung nach begleitenden oder folgenden Therapien im Beobachtungszeitraum schwierig.

Für die intraoperative, ultraschallgestützte Ablation bei teils größeren Läsionen findet sich ein medianes Überleben von 24 bis 29 Monaten bei großen Fallzahlen. Nach der radiologisch-interventionellen, perkutanen RFA sind bedingt vergleichbare Ergebnisse von 28 bis 36 Monaten dokumentiert, wobei diese Daten bei meist nicht resektablen Patienten erhoben wurden und eine negative Selektion vermutet werden darf [10,12-14].

Als Komplikationen der Radiofrequenzablation sind hitzeinduzierte Effekte auf benachbarte Organstrukturen zu nennen, u.a. Gallengangsstenosen, arteriovenöse Fisteln und Gefäßthrombosen. Daneben können alle typischen Ereignisse infolge einer Leberpunktion auftreten, darunter Blutungen und Infektionen. Die Häufigkeit der diesbezüglichen Majorkomplikationen wird mit 1,9-6\% angegeben. Eine spezielle Form der Nebenwirkung ist das Postablationssyndrom, welches bei etwa einem Drittel der Ablationen mit Fieber, Schwindel und Schmerzen nach 3 bis 5 Tagen auftritt. Als weitere Problematik wird die Induktion von Stichkanalmetastasen (Häufigkeit ca. 2,7\% in Abhängigkeit von der Tumorentität) diskutiert [34].

\section{Laserinduzierte Thermotherapie und weitere lokalablative Verfahren}

Bei der LITT wird durch Nd:YAG-Laser mit einer Wellenlänge von 1064 nm eine Hyperthermie erzeugt [35], die identisch zur Radiofrequenzablation wirkt und ebenso in der MR-Bildgebung überwacht werden kann [16]. Das Verfahren wurde insbesondere zur Anwendung im Kernspintomografen konzipiert, da anfangs keine MR-kompatiblen RFA-Sonden zur Verfügung standen und nur mit dieser Methode der erhöhte Weichteilkontrast und die Möglichkeit der Onlinethermometrie der MRT genutzt werden konnte. Mit der laserinduzierten Thermotherapie können zirkuläre Nekrosen mit einem Durchmesser von bis zu $5 \mathrm{~cm}$ (in Multiapplikatortechnik Erweiterung der Ablationszone bzw. Optimierung der Ablationsgeometrie möglich) erzeugt werden und der therapeutische Ansatz unterliegt damit denselben Beschränkungen wie denen der RFA (größere Tumoren, Nähe von kühlenden Gefäßen

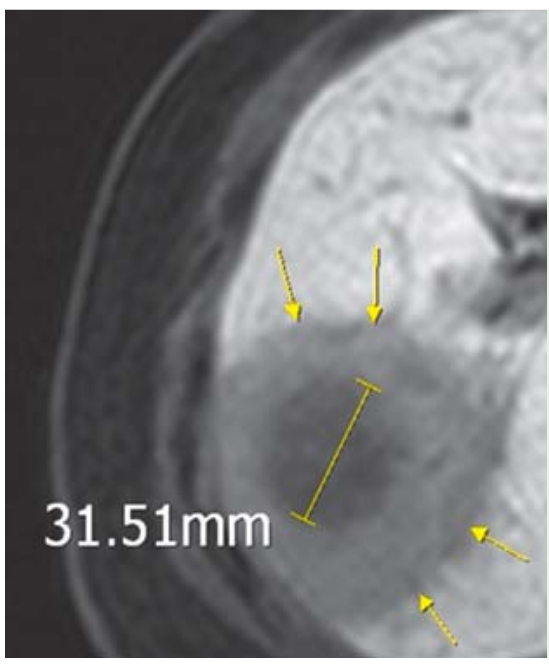

Abb. 4 MRT 3 Monate nach Brachytherapie: residuelle Nekrose der Metastase $\sim 3 \mathrm{~cm}$ deutlich hypointens (Messlinie), umgebende Strahlenreaktion mit strahleninduziertem Funktionsausfall des Leberparenchyms (fehlende Aufnahme des hepatozytenspezifischen Kontrastmittels Gd-EOB-DTPA, Pfeile).

oder hitzesensibler Strukturen) sowie ebenfalls vergleichbaren Komplikationen bei etwa 2,2\% der Patienten [35]. Nachteile der LITT sind die recht hohen Anschaffungskosten der Lasergeneratoren und die preisintensiven Applikatoren und Laserfasern.

Vor dem Hintergrund des Heat-Sink-Effekts könnte die Mikrowellenablation (MWA) eine geeignete Alternative zu LITT und RFA darstellen, da hierbei die Nachbarschaft zu größeren Gefäßen technisch nicht limitierend ist und gleichzeitig größere Ablationsvolumina möglich sind [36].

Für die laserinduzierte Thermotherapie sind zur Radiofrequenzablation vergleichbare 1-Jahres-Überlebensraten von über $90 \%$ an großen Patientenkollektiven publiziert, nach 5 Jahren leben jeweils noch ungefähr ein Drittel der Patienten. Das mediane Überleben ist mit 23 bis 42 Monaten angegeben [16,18].

Obwohl noch nicht an größeren Patientenzahlen für das kolorektale Karzinom validiert, sind auch nach der Mikrowellenablation 5-Jahres-Überlebensraten von bis zu 32\% möglich [37].

Ein weiteres vielversprechendes Verfahren stellt die irreversible Elektroporation dar. Bei dieser wird über eine Stromapplikation die Zellmembran der Zellen geschädigt und somit der Zelltod induziert. Erste klinische Untersuchungen zeigen sowohl die Machbarkeit als auch Verträglichkeit zur Ablation von Lebermetastasen [38]. Bisher fehlen jedoch Langzeiterfahrungen hinsichtlich der lokalen Kontrolle, des Überlebens und möglicher Komplikationen.

\section{Interstitielle Brachytherapie}

Als weiteres perkutan durchgeführtes Interventionsverfahren kann die Brachytherapie zur Tumormassereduktion und vollständigen Ablation bei Lebermetastasen eingesetzt werden, die aufgrund ihrer Größe nicht mehr einer RFA zugänglich sind. Darüber hinaus ergeben sich bei diesem Verfahren keine Einschränkungen durch Kühlungseffekte benachbarter Gefäße. Neben dem intraoperativen Einsatz (IORT) können unter Kontrolle durch CToder offene MRT-Systeme Bestrahlungskatheter in die Leberherde eingelegt werden. Nach Erstellen eines Planungs-CT/-MRT mit den einliegenden Kathetern erfolgt die Bestrahlungsplanung und anschließend die computergestützte Bestrahlung mittels einer Iridium-192-Quelle in Afterloading-Technik. Die bildgeführte Durchführung mittels CT oder MRT erlaubt hierbei eine komplikationsarme und genauere Katheterplatzierung und Bestrahlungsplanung im Vergleich zur intraoperativen Anwendung [39]. Gegenüber der perkutanen Bestrahlung mittels Teletherapie 

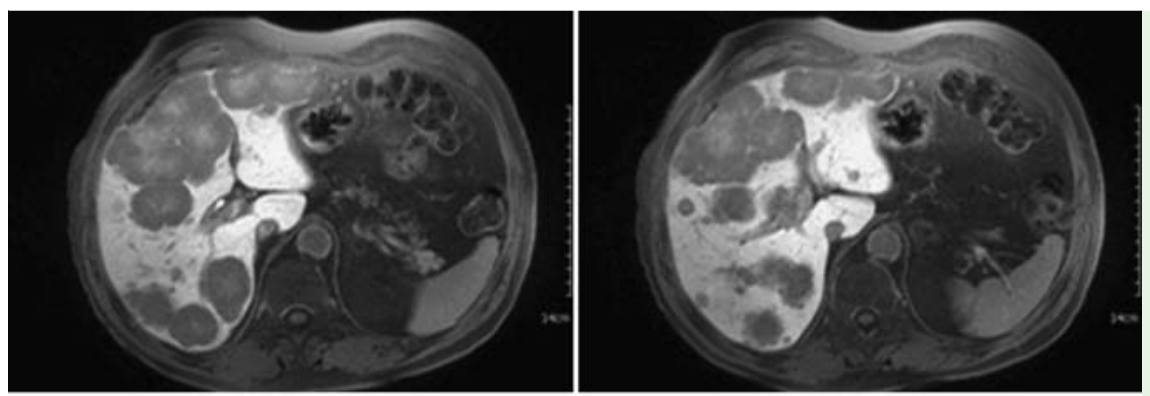

Abb. 5 MRT vor Radioembolisation: Ausgedehnte bilobäre Lebermetastasierung (T1w GRE mit
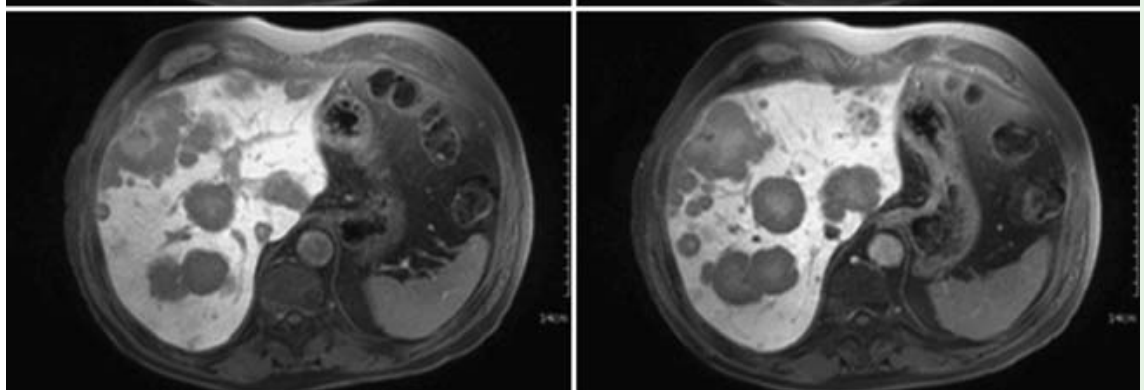

Gd-EOB-DTPA in der hepatobiliären Phase).
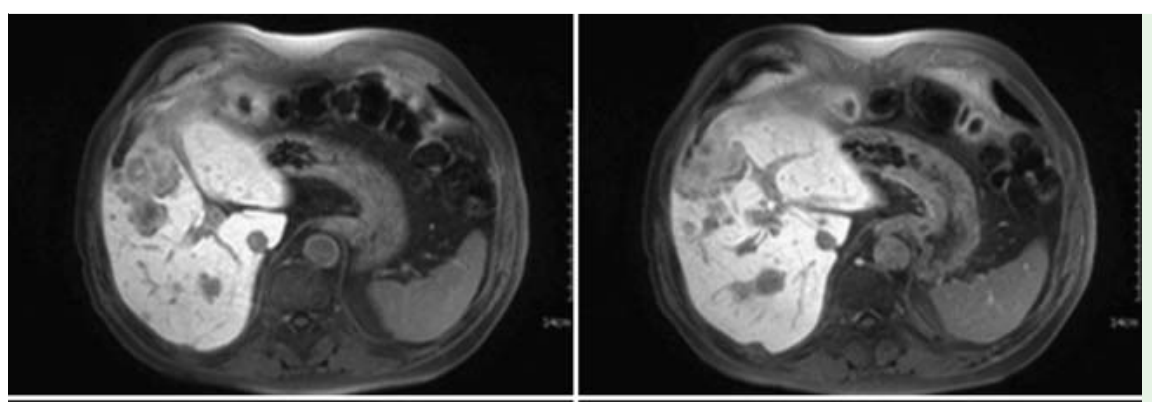

Abb. 6 MRT 3 Monate nach Radioembolisation: Partielle Remission der Lebermetastasen (T1w GRE mit Gd-EOB-DTPA in der hepatobiliären Phase).
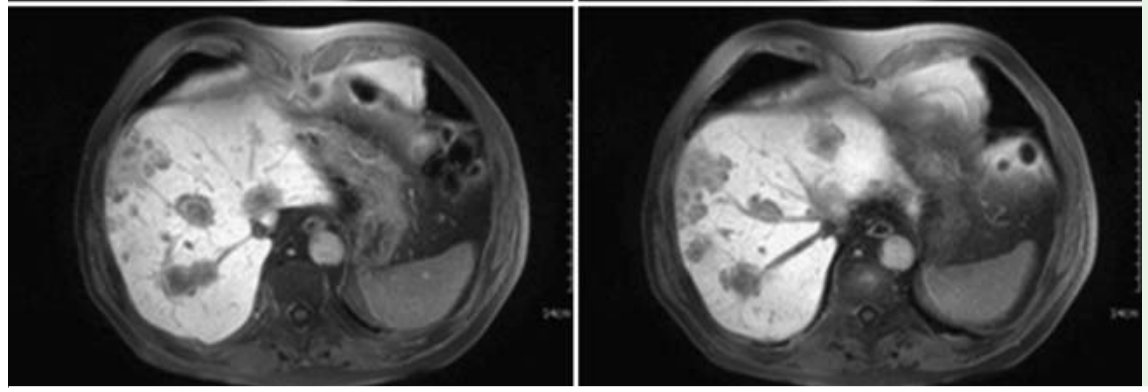

weist die interstitielle Brachytherapie zudem den Vorteil einer festen Platzierung der Strahlenquelle im Tumor ohne Beeinflussung durch Atemexkursionen auf [40]. Zur effektiven Ablation wird eine tumorumschließende Zieldosis von minimal 20 Gy angestrebt, wobei in den zentraleren Tumoranteilen sehr viel höhere Dosen erreicht werden [41].

Mit dem Verfahren der CT-gestützten Brachytherapie konnte für intensiv vortherapierte Patienten mit fortgeschrittener hepatischer Metastasierung eines kolorektalen Karzinoms (Größe 1,013,5 cm Durchmesser; medianer Diameter: $5 \mathrm{~cm}$ ) ein medianes Überleben von 23,4 Monaten aufgezeigt werden [21]. Anzumerken ist, dass die beobachteten Patienten einer negativen Selektion gegenüber den thermischen Verfahren unterliegen.

Die möglichen Komplikationen der Brachytherapie werden aktuell langzeitig untersucht, bislang noch nicht publizierte Daten zeigen eine Rate von 2,4\% an Majorkomplikationen. Neben den Punktionsrisiken durch teils mehrere Bestrahlungskatheter (Blutungen, Leberabszesse oder peritoneale Infektionen) ist vor allem eine strahleninduzierte, doch meist subklinische Schädigung des umliegenden Leberparenchyms zu beobachten, die durchschnittlich 6 Wochen nach der Therapie als verminderte Aufnahme hepatozytenspezifischer Kontrastmittel auffällt und die residuelle, häufig nekrotische Metastase umgibt [42].

- Abb. 3 und 4 zeigen als Beispiel eine präinterventionelle Lebermetastase sowie das Ansprechen 3 Monate nach der Therapie (nekrotische Metastase mit umgebender radiogener Schädigung des Leberparenchyms).

\section{Lokoregionäre Verfahren \\ $\nabla$}

\section{Yttrium-90-Radioembolisation}

Bei der Yttrium-90-Radioembolisation handelt es sich um ein Verfahren zur Behandlung disseminierter hepatischer Malignome, bei dem ein radioaktives Isotop, in diesem Falle ${ }^{90} \mathrm{Y}$, gebunden an Kunstharz- oder Glasmikrosphären, mittels eines Kathe- 
Tab. 1 Langzeitergebnisse nach interventioneller Therapie kolorektaler Lebermetastasen.

\begin{tabular}{|c|c|c|c|c|c|c|c|c|}
\hline \multirow[t]{2}{*}{ Autor } & \multirow{2}{*}{$\begin{array}{l}\text { Pat.- } \\
\text { Anzahl }\end{array}$} & \multicolumn{2}{|c|}{ Metastasen } & \multicolumn{5}{|l|}{ Ergebnisse } \\
\hline & & Anzahl & $\begin{array}{l}\text { Größe } \\
\text { (cm) }\end{array}$ & $\begin{array}{l}\text { medianes } \\
\text { Follow-up } \\
\text { (m) }\end{array}$ & $\begin{array}{l}\text { lokale } \\
\text { Kontrolle } \\
\text { (\%) }\end{array}$ & $\begin{array}{l}\text { Überlebens- } \\
\text { rate }(\%) \text {, } \\
(0,5 / 1 / 5 y)\end{array}$ & $\begin{array}{l}\text { Median } \\
\text { OS (m) }\end{array}$ & $\begin{array}{l}\text { Median } \\
\text { PFS (m) }\end{array}$ \\
\hline \multicolumn{9}{|l|}{ Radiofrequenzablation } \\
\hline Solbiati et al. 2001 [10] & 117 & 179 & $0,9-9,6$ & $6-52$ & 61 & $-\mid 93 /-$ & 36 & 12 \\
\hline Gillams et al. 2005 [11] & 167 & 1-27 p.P. & $1-12$ & - & - & $-/ 91 / 25$ & 31 & - \\
\hline Berber et al. 2005 [12]* & 135 & 1-12 p.P. & $1,2-10,2$ & - & - & - & 28,9 & - \\
\hline Siperstein et al. $2007[13]^{* *}$ & 234 & 292 & $<10,2$ & 24 & - & $-\mid-/ 18$ & 24 & - \\
\hline \multirow[t]{2}{*}{ Gillams et al. 2009 [14] } & 309 & $n<5$ p. $P$. & $<5$ & - & - & $-\mid-/ 18$ & 28 & - \\
\hline & & $n>5$ p.P. & $>5$ & - & - & $-1-13$ & 14 & - \\
\hline Sofocleous et al. 2011 [15] & 56 & 71 & $0,5-5,7$ & - & - & $-|91|-$ & 31 & - \\
\hline \multicolumn{9}{|l|}{ LITT } \\
\hline Mack et al. 2001 [16] & 393 & 1203 & $<5$ & $>18$ & - & $97 / 93 / 30$ & 42 & - \\
\hline Vogl et al. 2004 [17] & 603 & 1801 & $<5$ & $>18$ & - & $-/ 94 / 37$ & 35 & - \\
\hline Pech et al. 2007 [18] & 66 & 117 & $<5$ & 8,7 & - & $-1-1-$ & 23 & 6,1 \\
\hline \multicolumn{9}{|l|}{ Mikrowellenablation ${ }^{\# \#}$} \\
\hline Morita et al. 2004 [19] & 52 & - & - & - & - & $-1-120-24$ & - & - \\
\hline Ogata et al. 2008 [20] & 50 & - & 2,1 & 33 & - & $-1-132$ & - & - \\
\hline \multicolumn{9}{|l|}{ interstit. Brachytherapie } \\
\hline Ricke et al. 2010 [21] & 73 & 199 & $1-13,5$ & 41 & 75 & - & 23,4 & 6 \\
\hline Radioembolisation & & Tumorlast & & & & & & \\
\hline Stubbs et al. $2006[22]^{* * *}$ & 80 & - & & 3 & 83 & - & - & - \\
\hline Kennedy et al. 2006 [23] & 208 & - & & 13 & - & - & $10,5^{\#}$ & - \\
\hline Jakobs et al. 2008 [24] & 41 & - & & - & - & - & 10,5 & - \\
\hline Cosimelli et al. 2010 [25] & 50 & $<25-50 \%$ & & 11 & - & $-|50|-$ & 12,6 & 3,7 \\
\hline Seidensticker et al. 2011 [26] & 29 & $20-50 \%$ & & - & - & - & 8,3 & 5,5 \\
\hline Kosmider et al. $2011[27]^{* * *}$ & 19 & - & & 18,6 & - & - & 29,4 & 10,4 \\
\hline
\end{tabular}

* laparoskopische RFA; ** laparoskopische RFA nach Hemihepatektomie; *** Kombinationsregime mit Chemotherapie (5-FU intraarteriell o. systemisch); \# Überlebensangabe nur für Therapieresponder; \#\# gemischte Kollektive mit offener/perkutaner MWA bzw. MWA und RFA, aus Boutros et al. 2010 [37]; OS: Overall survival; PFS: progressionsfreies Überleben.

ters in die leberversorgenden Arterien appliziert wird. Die Katheterpositionierung erfolgt dabei mittels digitaler Subtraktionsangiografie über einen transfemoralen Zugang. Maligne Prozesse der Leber werden bis zu 90\% über Arterien versorgt, während gesundes Leberparenchym hauptsächlich über die V. portae den Blutzustrom erhält [43]. Damit kann durch die Radioembolisation sowohl die Blutversorgung von Tumoren unterbunden werden als auch eine Bestrahlung derselben durchgeführt werden. Vor der Therapie wird eine digitale Subtraktionsangiografie zur Beurteilung der Gefäßsituation der Leber durchgeführt. Kollaterale Arterien, die einen potenziellen extrahepatischen Abstrom der applizierten Mikrosphären in den Gastrointestinaltrakt ermöglichen, werden dabei mittels thrombogener Metallspiralen (Coils) verschlossen [44]. Anschließend wird 99-mT-markiertes makroaggregiertes Albumin in die Leber appliziert, um in einer nachfolgenden Szintigrafie eine Anreicherung im extrahepatischen Oberbauch auszuschließen und den Leber-Lungen-Shunt für die Dosisberechnung zu quantifizieren [45]. Erst wenn diese Simulation erfolgreich verläuft, kann in einer weiteren Sitzung das therapeutisch wirksame Radioembolisat appliziert werden. In der ersten prospektiven Evaluation dieses Verfahrens als Monotherapie bei Patienten mit fortgeschrittenem, hepatisch metastasiertem kolorektalen Karzinom und weitestgehend ausgeschöpften Therapieoptionen zeigte sich ein medianes Gesamtüberleben von 12,6 Monaten, das 2-Jahres-Überleben erreichte ein Fünftel der eingeschlossenen Patienten [25]. Gray et al. zeigten in einer randomisierten Studie, in der die Radioembolisation in Kombination mit einer intraarteriell applizierten Chemotherapie mittels Floxuridin angewendet wurde, ein 2-Jahres-Überleben von 39\% gegenüber 29\% nach alleiniger Chemotherapie [46].
Das Beispiel einer disseminierten Lebermetastasierung zeigen $\checkmark$ Abb. 5 vor der Radioembolisation und 0 Abb. 6 in der Nachsorge nach 3 Monaten.

Aufgrund der Anwendung in interdisziplinären Therapiekonzepten ist die Bewertung der erreichten Endpunkte schwierig und es fehlen Daten zur alleinigen Effektivität der Radioembolisation. In der Salvage-Situation kann ein medianes Überleben von 8 bis 12 Monaten erreicht werden im Vergleich zu Best Supportive Care (BSC), worunter nur 3,5 Monate zu verzeichnen sind [23,24,26]. Kommt die Yttrium-90-Radioembolisation jedoch in einem früheren Krankheitsstadium in Kombination mit einer systemischen Chemotherapie zum Einsatz, sind Überlebenszeiten bis zu 30 Monate möglich [27]. In einem großen multizentrischen Studienrahmen wird aktuell prospektiv-randomisiert die Wirksamkeit der Yttrium-90-Radioembolisation in Kombination mit FOLFOX als Erstlinientherapie untersucht.

Obwohl mit einer geringen Morbidität assoziiert, zeigen sich bei der Radioembolisation zumeist subklinische strahleninduzierte Leberfunktionsstörungen, besonders wenn die gesamte Leber in einer Therapiesitzung behandelt wird. Zu den schwereren Komplikationen zählen neben Pleuraergüssen vor allem Aszites, Bilirubinerhöhung sowie Ikterus, die unter der Bezeichnung der „radioembolization-induced liver disease“ zusammengefasst werden und mit einer Häufigkeit von etwa $10 \%$ auftreten und durch eine zweiseitige Therapie der Leber weiter gesenkt werden können [47]. Größere Komplikationen durch die katheterangiografische Technik sind bei sorgfältiger Durchführung dagegen nicht zu erwarten. 


\section{Diskussion}

\section{V}

Die minimalinvasive interventionell-radiologische Therapie kolorektaler Lebermetastasen zielt auf eine möglichst belastungsarme Behandlung des Patienten bei hoher Effektivität. Dabei bietet sie für verschiedene Tumorkonfigurationen eine geeignete Methode von perkutan interstitieller Ablation eines oligonodulären Befalls bis zur Therapie einer diffusen hepatischen Filiarisierung über den transarteriellen Zugang. Nachteilig mag die lokale bzw. lokoregionäre Begrenzung der Therapie erscheinen, doch die häufig prognostisch führende Lebermetastasierung kann oftmals gut kontrolliert werden.

Bestehen keine Kontraindikationen für eine chirurgische Resektion, gilt diese unverändert als Goldstandard und wird allgemein präferiert, obwohl der potenziell kurative Charakter für viele Patienten oftmals nicht langfristig besteht. Unbestritten ist dabei die Prognoseverbesserung durch die R0-Resektion [48]. Mit den neueren Verfahren der atypischen Resektion können immer mehr Patienten einer operativen Therapie zugeführt werden, jedoch wurden hierbei in einer großen Studie nahezu 10\% an R1Resektionen nachgewiesen [49]. Dem vergleichbar sind die Lokalrezidivquoten nach Radiofrequenzablation mit 3,6 bis $16 \%$ (Metastasen $<3 \mathrm{~cm}$ ) $[50,51]$.

Das 5-Jahres-Überleben nach bildgeführter Radiofrequenzablation bei kolorektalen Lebermetastasen kann bis zu 33\% betragen und ist mit den Überlebensraten in den bereits genannten chirurgischen Konzepten durchaus vergleichbar, wobei es zu beachten gilt, dass es sich bei der RFA hier um selektierte Patientenkollektive handelt ( $<3$ Tumoren; Diameter: $<3,5 \mathrm{~cm}$ ) [14]. Die laparoskopisch durchgeführte Radiofrequenzablation zeigt zudem ähnliche Ergebnisse, wobei die operativ durchgeführte RFA in der Regel einer positiven Patientenselektion unterliegt [12,52]. Hier lässt sich auch feststellen, dass weder die Tumoranzahl (< oder $>3$ ) noch das Vorliegen extrahepatischer Manifestationen einen Einfluss auf das Überleben haben. Nur der maximale Durchmesser der dominanten Läsion $<3 \mathrm{~cm}$ ist als prognostisch positiv $\mathrm{zu}$ bewerten [12].

Eine Metaanalyse von 7 nicht randomisierten Studien bei solitären Lebermetastasen zeigte wiederum einen signifikanten Überlebensvorteil für die Patienten mit hepatischer Resektion im Vergleich zur RFA, weshalb die Empfehlung bei Operabilität weiterhin zur Resektion geht [53].

Ein weiterer interessanter Ansatz ist das „Test-of-time“-Verfahren, bei dem potenziell kurativ zu resezierende Lebermetastasen zunächst einer bildgeführten Radiofrequenzablation unterzogen werden und die Operation ca. 6-12 Wochen später im extra- und intrahepatisch rezidivfreien Intervall geplant ist. Studien zeigen, dass 70\% der Patienten innerhalb des Beobachtungszeitraums vor der geplanten Operation einen extra- oder intrahepatischen Progress außerhalb der therapierten Läsion aufwiesen, wodurch festzustellen ist, dass diese Patienten wahrscheinlich nicht von einer initialen Resektion nachhaltig profitiert hätten [54,55].

Perspektivisch sind die Anwendungen der Radiofrequenzablation über den bisher rein palliativen Charakter hinaus durchaus denkbar und als Alternative oder zusätzlich zur chirurgischen Resektion vorstellbar [56].

Die laserinduzierte Thermotherapie zeigt bei ähnlicher Indikation vergleichbare Ergebnisse, wobei gesagt werden muss, dass hierzu keine prospektiven Analysen vorliegen. Auch die Mikrowellenablation mit ihren Vorteilen hinsichtlich der Unabhängigkeit von Kühlungseffekten und Größenlimitationen schafft zur RFA vergleichbare Resultate mit einem 5-Jahres-Überleben bis
$32 \%$, doch sind bisher teils Patientenkollektive zusammen mit der RFA und insgesamt keine größeren Fallzahlen für das kolorektale Karzinom publiziert [37].

Wenn speziell die Tumorgröße oder -lokalisation die thermoablativen Verfahren technisch limitiert, stellt die interstitielle Brachytherapie eine lohnende Erweiterung des interventionellradiologischen Spektrums dar und wird in enger Zusammenarbeit mit einem Strahlentherapeuten in Afterloading-Technik durchgeführt. Für Lebermetastasen bis zu 13,5 cm Durchmesser kann mit der interstitiellen Brachytherapie eine lokale Kontrolle bei 75\% der Läsionen erreicht werden (Zieldosis: 15-25 Gy). In einer Subgruppenanalyse zeigte sich eine signifikant bessere Kontrolle von $84 \%$, wenn tumorumschließend eine Dosis von mindestens 20 Gy appliziert wird. Auch Patienten mit größeren Tumorvolumina $(>5 \mathrm{~cm}$ ) zeigten ein zur thermoablativen Therapie vergleichbares medianes Überleben von 23,4 Monaten [21]. Auch bei Vorliegen einer multifokalen, diffusen Metastasierung kann die radiologische Mikrotherapie eine wertvolle Ergänzung zur oftmals belastenden systemischen Chemotherapien darstellen, indem mit Verfahren wie z.B. der Yttrium-90-Radioembolisation mit geringem Morbiditätsrisiko Tumormassen reduziert oder zumindest kontrolliert werden können. Zugute kommt dabei die hohe Dosis, die am Tumor erreicht werden kann und auch bei chemorefraktären Tumoren noch eine Wirksamkeit aufweist. Die besten Ergebnisse konnten dennoch dann gezeigt werden, wenn Verfahren wie die Radioembolisation in einem frühen Stadium synergistisch mit einer systemischen oder intraarteriellen Chemotherapie zur Anwendung kommen [22,27].

Aber auch in der Salvage-Therapie profitieren Patienten von der Radioembolisation, wie in zahlreichen Studien gezeigt wurde [23, 24, 26].

Zudem sind in jüngerer Zeit die ersten Studien zur Anwendung der transarteriellen Chemoembolisation mit zytostatikabeladenen Embolisationspartikeln (z.B. Irinotecan) veröffentlicht, erste Phase-II-Studien zeigen ein gutes Ansprechen mit einem medianen Überleben von 25 Monaten beim leberdominant metastasierten kolorektalen Karzinom. Jedoch fehlen auch hier randomisierte Studien mit einem Vergleich zu etablierten systemischen Therapien [57].

Unbefriedigend bleibt die insgesamt unzureichende Evidenzlage bei den meisten interventionellen Verfahren, weshalb die Durchführung kontrollierter randomisierter Studien kurzfristiges Ziel für die verschiedenen Methoden sein sollte.

Ein abschließender Aspekt sollte die interdisziplinäre Zusammenarbeit der onkologisch tätigen Fachrichtungen sein, von der Patienten mit Lebermetastasen deutlich profitieren können. Neben der engen Verflechtung von chirurgischer Resektion und systemischer Therapie zeigt sich auch beispielsweise in der Kombination von der Radiofrequenzablation mit der Resektion oder Chemotherapie ein den jeweils einzelnen Modalitäten überlegenes Gesamtüberleben [58]. Dies wird ebenfalls in der Kombination aus systemischer Therapie und wiederholten interstitiellen Brachytherapien deutlich [21].

Interessenkonflikt: Nein

\section{Literatur}

1 Cady B, Monson DO, Swinton NW. Survival of patients after colonic resection for carcinoma with simultaneous liver metastases. Surg Gynecol Obstet 1970; 131: 697-700

2 Wood $C B$, Gillis $C R$, Blumgart $L H$. A retrospective study of the natural history of patients with liver metastases from colorectal cancer. Clin Oncol 1976; 2: 285-288 
3 Hompes D, Prevoo W, Ruers T. Radiofrequency ablation as a treatment tool for liver metastases of colorectal origin. Cancer Imaging 2011; 11: 23-30

4 Ito K, Govindarajan A, Ito $\mathrm{H}$ et al. Surgical treatment of hepatic colorectal metastasis: evolving role in the setting of improving systemic therapies and ablative treatments in the 21st century. Cancer J 2010; 16: $103-110$

5 Andreou A, Brouquet A, Abdalla EK et al. Repeat hepatectomy for recurrent colorectal liver metastases is associated with a high survival rate. HPB (Oxford) 2011; 13: 774-782

6 Khatri VP, Petrelli NJ, Belghiti J. Extending the frontiers of surgical therapy for hepatic colorectal metastases: is there a limit? J Clin Oncol 2005; 23: 8490-8499

7 Goldberg RM, Sargent DJ, Morton RF et al. A randomized controlled trial of fluorouracil plus leucovorin, irinotecan, and oxaliplatin combinations in patients with previously untreated metastatic colorectal cancer. J Clin Oncol 2004; 22: 23-30

8 Yothers G. Toward progression-free survival as a primary end point in advanced colorectal cancer. J Clin Oncol 2007; 25: 5153-5154

9 Sutherland LM, Williams JA, Padbury RT et al. Radiofrequency ablation of liver tumors: a systematic review. Arch Surg 2006; 141: 181-190

10 Solbiati L, Livraghi T, Goldberg SN et al. Percutaneous radio-frequency ablation of hepatic metastases from colorectal cancer: long-term results in 117 patients. Radiology 2001; 221: 159-166

11 Gillams AR, Lees WR. Radiofrequency ablation of colorectal liver metastases. Abdom Imaging 2005; 30: 419-426

12 Berber E, Pelley R, Siperstein AE. Predictors of survival after radiofrequency thermal ablation of colorectal cancer metastases to the liver: a prospective study. J Clin Oncol 2005; 23: 1358-1364

13 Siperstein $A E$, Berber E, Ballem $N$ et al. Survival after radiofrequency ablation of colorectal liver metastases: 10-year experience. Ann Surg 2007; 246: 559-565

14 Gillams AR, Lees WR. Five-year survival in 309 patients with colorectal liver metastases treated with radiofrequency ablation. Eur Radiol 2009; 19: 1206-1213

15 Sofocleous CT, Petre EN, Gonen M et al. CT-guided radiofrequency ablation as a salvage treatment of colorectal cancer hepatic metastases developing after hepatectomy. J Vasc Interv Radiol 2011; 22: 755-761

16 Mack MG, Straub R, Eichler K et al. Percutaneous MR imaging-guided laser-induced thermotherapy of hepatic metastases. Abdom Imaging 2001; 26: 369-374

17 Vogl TJ, Straub R, Eichler K et al. Colorectal carcinoma metastases in liver: laser-induced interstitial thermotherapy-local tumor control rate and survival data. Radiology 2004; 230: 450-458

18 Pech M, Wieners G, Freund T et al. MR-guided interstitial laser thermotherapy of colorectal liver metastases: efficiency, safety and patient survival. Eur J Med Res 2007; 12: 161-168

19 Morita T, Shibata T, Okuyama $M$ et al. [Microwave coagulation therapy for liver metastases from colorectal cancer]. Gan To Kagaku Ryoho 2004; 31: 695-699

20 Ogata Y, Uchida S, Hisaka $T$ et al. Intraoperative thermal ablation therapy for small colorectal metastases to the liver. Hepatogastroenterology 2008; 55: 550-556

21 Ricke J, Mohnike K, Pech $M$ et al. Local response and impact on survival after local ablation of liver metastases from colorectal carcinoma by computed tomography-guided high-dose-rate brachytherapy. Int J Radiat Oncol Biol Phys 2010; 78: 479-485

22 Stubbs RS, O'Brien I, Correia MM. Selective internal radiation therapy with $90 \mathrm{Y}$ microspheres for colorectal liver metastases: single-centre experience with 100 patients. ANZ J Surg 2006; 76: 696-703

23 Kennedy AS, Coldwell D, Nutting C et al. Resin 90Y-microsphere brachytherapy for unresectable colorectal liver metastases: modern USA experience. Int J Radiat Oncol Biol Phys 2006; 65: 412-425

24 Jakobs TF, Hoffmann RT, Dehm K et al. Hepatic yttrium-90 radioembolization of chemotherapy-refractory colorectal cancer liver metastases. J Vasc Interv Radiol 2008; 19: 1187-1195

25 Cosimelli M, Golfieri R, Cagol PP et al. Multi-centre phase II clinical trial of yttrium-90 resin microspheres alone in unresectable, chemotherapy refractory colorectal liver metastases. Br J Cancer 2010; 103: 324-331

26 Seidensticker R, Denecke T, Kraus $P$ et al. Matched-pair comparison of radioembolization plus best supportive care versus best supportive care alone for chemotherapy refractory liver-dominant colorectal metastases. Cardiovasc Intervent Radiol 2011; 35: 1066-1073
27 Kosmider S, Tan TH, Yip D et al. Radioembolization in combination with systemic chemotherapy as first-line therapy for liver metastases from colorectal cancer. J Vasc Interv Radiol 2011; 22: 780-786

28 Pereira PL, Trubenbach J, Schmidt D. Radiofrequency ablation: basic principles, techniques and challenges. Rofo 2003; 175: 20-27

29 Pereira PL. Perkutane bildgesteuerte Leber-Tumortherapie mittels Radiofrequenz-Ablation (RFA). Deutsche Gesellschaft für Interventionelle Radiologie und minimal-invasive Therapie 2012. Im Internet: http://v2.degir.de/download/dokman/Leitlinie-RFA; Stand 9.2.2012

$30 \mathrm{Kim} \mathrm{KH}$, Yoon YS, Yu CS et al. Comparative analysis of radiofrequency ablation and surgical resection for colorectal liver metastases. J Korean Surg Soc 2011; 81: 25-34

31 Jung EM, Clevert DA, Rupp N. Contrast-enhanced ultrasound with Optison in percutaneous thermoablation of liver tumors. Rofo 2003; 175: $1403-1412$

32 Stroszczynski C, Gretschel S, Gaffke G et al. [Laser-induced thermotherapy (LITT) for malignant liver tumours: the role of sonography in catheter placement and observation of the therapeutic procedure]. Ultraschall Med 2002; 23: 163-167

33 Rempp H, Clasen S, Pereira PL. Image-based monitoring of magnetic resonance-guided thermoablative therapies for liver tumors. Cardiovasc Intervent Radiol 2011 Jul 22 [Epub ahead of print]

34 Nemcek AA. Complications of radiofrequency ablation of neoplasms. Semin Intervent Radiol 2006; 23: 177-187

$35 \mathrm{Vogl} \mathrm{TJ}$, Straub R, Zangos S et al. MR-guided laser-induced thermotherapy (LITT) of liver tumours: experimental and clinical data. Int J Hyperthermia 2004; $20: 713-724$

36 Simon CJ, Dupuy DE, Iannitti DA et al. Intraoperative triple antenna hepatic microwave ablation. AJR Am J Roentgenol 2006; 187: W333W340

37 Boutros C, Somasundar P, Garrean S et al. Microwave coagulation therapy for hepatic tumors: review of the literature and critical analysis. Surg Oncol 2010; 19: e22-e32

38 Thomson KR, Cheung W, Ellis SJ et al. Investigation of the safety of irreversible electroporation in humans. J Vasc Interv Radiol 2011; 22: 611 621

39 Dritschilo A, Harter KW, Thomas D et al. Intraoperative radiation therapy of hepatic metastases: technical aspects and report of a pilot study. Int J Radiat Oncol Biol Phys 1988; 14: 1007-1011

40 Herfarth KK, Debus J, Lohr F et al. Stereotactic single-dose radiation therapy of liver tumors: results of a phase I/II trial. J Clin Oncol 2001; 19: $164-170$

41 Ricke J, Wust P, Stohlmann A et al. CT-Guided brachytherapy. A novel percutaneous technique for interstitial ablation of liver metastases. Strahlenther Onkol 2004; 180: 274-280

42 Seidensticker $M$, Seidensticker $R$, Mohnike $K$ et al. Quantitative in vivo assessment of radiation injury of the liver using Gd-EOB-DTPA enhanced MRI: Tolerance dose of small liver volumes. Radiat Oncol 2011; 6: 40

43 Wallace S, Carrasco CH, Charnsangavej C et al. Hepatic artery infusion and chemoembolization in the management of liver metastases. Cardiovasc Intervent Radiol 1990; 13: 153-160

44 Liu DM, Salem R, Bui JT et al. Angiographic considerations in patients undergoing liver-directed therapy. J Vasc Interv Radiol 2005; 16: 911935

45 Denecke T, Ruhl R, Hildebrandt B et al. Planning transarterial radioembolization of colorectal liver metastases with Yttrium 90 microspheres: evaluation of a sequential diagnostic approach using radiologic and nuclear medicine imaging techniques. Eur Radiol 2008; 18: 892-902

46 Gray B, Van Hazel G, Hope M et al. Randomised trial of SIR-Spheres plus chemotherapy vs. chemotherapy alone for treating patients with liver metastases from primary large bowel cancer. Ann Oncol 2001; 12: $1711-1720$

47 Seidensticker R, Seidensticker M, Damm R et al. Hepatic toxicity after radioembolization of the liver using (90)Y-microspheres: sequential lobar versus whole liver approach. Cardiovasc Intervent Radiol 2011; 35: $1109-1118$

48 Scheele J, Altendorf-Hofmann A, Grube Tet al. Resection of colorectal liver metastases. What prognostic factors determine patient selection? Chirurg 2001; 72: 547-560

49 Welsh FK, Tekkis PP, O'Rourke T et al. Quantification of risk of a positive (R1) resection margin following hepatic resection for metastatic colorectal cancer: an aid to clinical decision-making. Surg Oncol 2008; 17: $3-13$ 
50 Berber E, Siperstein A. Local recurrence after laparoscopic radiofrequency ablation of liver tumors: an analysis of 1032 tumors. Ann Surg Oncol 2008; 15: 2757-2764

51 Mulier S, Ni Y, Jamart J et al. Local recurrence after hepatic radiofrequency coagulation: multivariate meta-analysis and review of contributing factors. Ann Surg 2005; 242: 158-171

52 Van Tilborg AA, Meijerink MR, Sietses C et al. Long-term results of radiofrequency ablation for unresectable colorectal liver metastases: a potentially curative intervention. Br J Radiol 2011; 84: 556-565

53 Wu YZ, Li B, Wang T et al. Radiofrequency ablation vs. hepatic resection for solitary colorectal liver metastasis: A meta-analysis. World J Gastroenterol 2011; 17: 4143-4148

54 Lambert LA, Colacchio TA, Barth jr. RJ. Interval hepatic resection of colorectal metastases improves patient selection. Arch Surg 2000; 135: 473-479
55 Livraghi T, Solbiati L, Meloni F et al. Percutaneous radiofrequency ablation of liver metastases in potential candidates for resection: the "testof-time approach". Cancer 2003; 97: 3027-3035

$56 \mathrm{Kim} \mathrm{KH}$, Yoon YS, Yu CS et al. Comparative analysis of radiofrequency ablation and surgical resection for colorectal liver metastases. J Korean Surg Soc 2011; 81: 25-34

57 Aliberti C, Fiorentini G, Muzzio PC et al. Trans-arterial chemoembolization of metastatic colorectal carcinoma to the liver adopting DC Bead ${ }^{\circledR}$, drug-eluting bead loaded with irinotecan: results of a phase II clinical study. Anticancer Res 2011; 31: 4581-4587

58 Stang A, Fischbach $R$, Teichmann $W$ et al. A systematic review on the clinical benefit and role of radiofrequency ablation as treatment of colorectal liver metastases. Eur J Cancer 2009; 45: 1748-1756 